\title{
Determination of the differential expression of mitochondrial long non-coding RNAs as a noninvasive diagnosis of bladder cancer
}

Alexis Rivas ${ }^{1,2}$, Verónica Burzio ${ }^{1,2}$, Eduardo Landerer ${ }^{3,4}$, Vincenzo Borgna ${ }^{1,3}$, Sebastian Gatica', Rodolfo Ávila ${ }^{1,2}$, Constanza López ${ }^{1}$, Claudio Villota ${ }^{1,2}$, Rodrigo de la Fuente ${ }^{5}$, Javiera Echenique ${ }^{1}$, Luis O Burzio ${ }^{1,2}$ and Jaime Villegas ${ }^{1,2^{*}}$

\begin{abstract}
Background: Bladder cancer is a significant cause of morbidity and mortality with a high recurrence rate. Early detection of bladder cancer is essential in order to remove the tumor, to preserve the organ and to avoid metastasis. The aim of this study was to analyze the differential expression of mitochondrial non-coding RNAs (sense and antisense) in cells isolated from voided urine of patients with bladder cancer as a noninvasive diagnostic assay.

Methods: The differential expression of the sense (SncmtRNA) and the antisense (ASncmtRNAs) transcripts in cells isolated from voided urine was determined by fluorescent in situ hybridization. The test uses a multiprobe mixture labeled with different fluorophores and takes about 1 hour to complete. We examined the expression of these transcripts in cells isolated from urine of 24 patients with bladder cancer and from 15 healthy donors.

Results: This study indicates that the SncmtRNA and the ASncmtRNAs are stable in cells present in urine. The test reveals that the expression pattern of the mitochondrial transcripts can discriminate between normal and tumor cells. The analysis of 24 urine samples from patients with bladder cancer revealed expression of the SncmtRNA and down-regulation of the ASncmtRNAs. Exfoliated cells recovered from the urine of healthy donors do not express these mitochondrial transcripts. This is the first report showing that the differential expression of these mitochondrial transcripts can detect tumor cells in the urine of patients with low and high grade bladder cancer.

Conclusion: This pilot study indicates that fluorescent in situ hybridization of cells from urine of patients with different grades of bladder cancer confirmed the tumor origin of these cells. Samples from the 24 patients with bladder cancer contain cells that express the SncmtRNA and down-regulate the ASncmtRNAs. In contrast, the hybridization of the few exfoliated cells recovered from healthy donors revealed no expression of these mitochondrial transcripts. This assay can be explored as a non-invasive diagnostic tool for bladder cancer.
\end{abstract}

\section{Background}

Bladder cancer $(\mathrm{BC})$ is an important cause of morbidity and mortality, with an estimated 386.000 new cases and 150.000 deaths occurring worldwide in 2008 [1]. Bladder tumors are classified into four categories: papilloma,

\footnotetext{
* Correspondence: jvillegas@bioschile.cl

${ }^{1}$ Andes Biotechnologies S.A. and Fundación Ciencia para la Vida, 7780272

Santiago, Chile

${ }^{2}$ Departamento de Ciencia Biológicas, Facultad de Ciencias Biológicas,

Universidad Andrés Bello, 8370146 Santiago, Chile

Full list of author information is available at the end of the article
}

low-grade carcinoma, high-grade carcinoma and carcinoma in situ [2]. About $90 \%$ of bladder cancers are urothelial carcinomas and transitional cell carcinomas (TCC) and the rest include squamous cell carcinomas and adenocarcinomas. As many other types of cancer, early detection of $\mathrm{BC}$ will allow effective treatments of patients, improving long-term survival.

The "gold standard" in the detection of BC is cystoscopy. This examination, however, is unpleasant, time consuming, expensive and may result in infections and urethral damage [3]. On the other hand, urine cytology

\section{Biomed Central}

(c) 2012 Rivas et al.; licensee BioMed Central Ltd. This is an Open Access article distributed under the terms of the Creative Commons Attribution License (http://creativecommons.org/licenses/by/2.0), which permits unrestricted use, distribution, and reproduction in any medium, provided the original work is properly cited. 
has high specificity but low sensitivity, especially in lowgrade disease $[4,5]$. To improve the detection of BC cells in voided urine, several tumor markers and tests have been developed [6,7]. One of these tests is based on fluorescent in situ hybridization (FISH) to detect chromosomal alterations characteristic of $\mathrm{BC}$ [8].

Human cells express a family of mitochondrial long non-coding RNAs (ncRNA) containing stem-loop structures. One of these transcripts, the sense mitochondrial ncRNA or SncmtRNA, is expressed in normal proliferating cells and tumor cells but not in non-dividing cells $[9,10]$. Experimental evidences suggest that this transcript plays a regulatory role of the cell cycle [11]. In addition, normal human proliferating cells in culture or in normal human tissues express two antisense transcripts, AsncmtRNA-1 and AsncmtRNA-2 [10]. Interestingly, the SncmtRNA and the AsncmtRNAs exit the mitochondria and localize to the cytoplasm and the nucleus in association with chromatin and nucleoli, suggesting that the function of these transcripts take place outside the organelle [12].

The function of the ASncmtRNAs is less clear. However, an interesting observation is that the ASncmtRNAs are down-regulated in tumor cell lines as well as in tumor cells present in different types of human cancer and patients [10]. In situ hybridization of twelve BC biopsies from different patients shows expression of the SncmtRNA and down-regulation of the ASncmtRNAs [10]. Since down-regulation of the ASncmtRNAs seems to be independent of the tissue of origin of tumor cells, the differential expression of these transcripts can be applied as a cancer diagnostic method for cells in suspension. Here, we present a one-tube fluorescence in situ hybridization protocol applied to cells in suspension (S-FISH), that takes about $60 \mathrm{~min}$ to perform and using simultaneously labeled probes for both SncmtRNA and AsncmtRNAs. This method was applied to cells isolated from urine of patients with bladder cancer (BC). In twenty four patients with low and high grade of $\mathrm{BC}$, S-FISH revealed cells expressing the SncmtRNAs and not the ASncmtRNAs, hence corresponding to cancer

Table 1 Distribution of tumor stage and grade among all patients included in this study

\begin{tabular}{lccccc}
\hline Grade and stage & \multicolumn{5}{c}{} \\
\cline { 2 - 6 } distribution & None & Grade $\mathbf{1}$ & Grade 2 & Grade 3 & Total \\
\hline No tumor & 15 & & & & 15 \\
Ta & & 11 & 2 & 4 & 17 \\
T1 & & 1 & 1 & 1 & 3 \\
T2-4 & & & & 3 & 3 \\
CIS & & & & 1 & 1 \\
Total & $\mathbf{1 5}$ & $\mathbf{1 2}$ & $\mathbf{3}$ & $\mathbf{9}$ & $\mathbf{3 9}$ \\
\hline
\end{tabular}

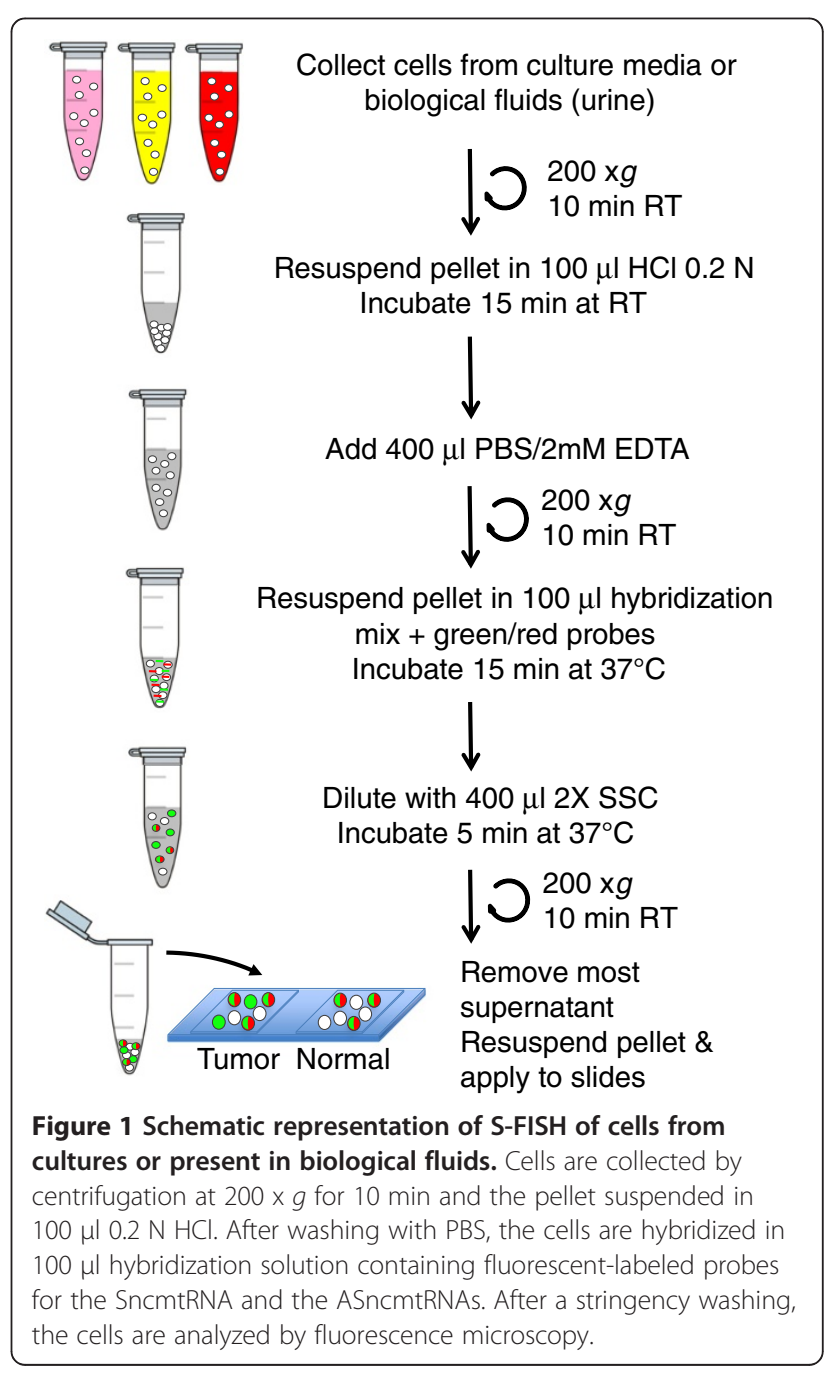

cells phenotype. The expression of these transcripts was negative in the few cells isolated from the urine of healthy donors. The differential expression of the SncmtRNA and the ASncmtRNAs in cells isolated from voided urine can be explored as a new non-invasive diagnostic test for $\mathrm{BC}$.

\section{Methods}

\section{Tumor cell culture}

T24 and RT4 cells (human bladder carcinoma) and DU-145 cells (prostate carcinoma) were cultured according to ATCC recommendations. Cultures were maintained in a humidified incubator at $37^{\circ} \mathrm{C}$ and $5 \% \mathrm{CO}_{2}$. Peripheral blood mononuclear cells (PBMC) from healthy donors were isolated and stimulated with phytohaemagglutinin (PHA) for $48 \mathrm{~h}$ as described before [9,10,13]. Primary renal mixed epithelial cells were obtained from ATCC and cultured according to ATCC guidelines. 


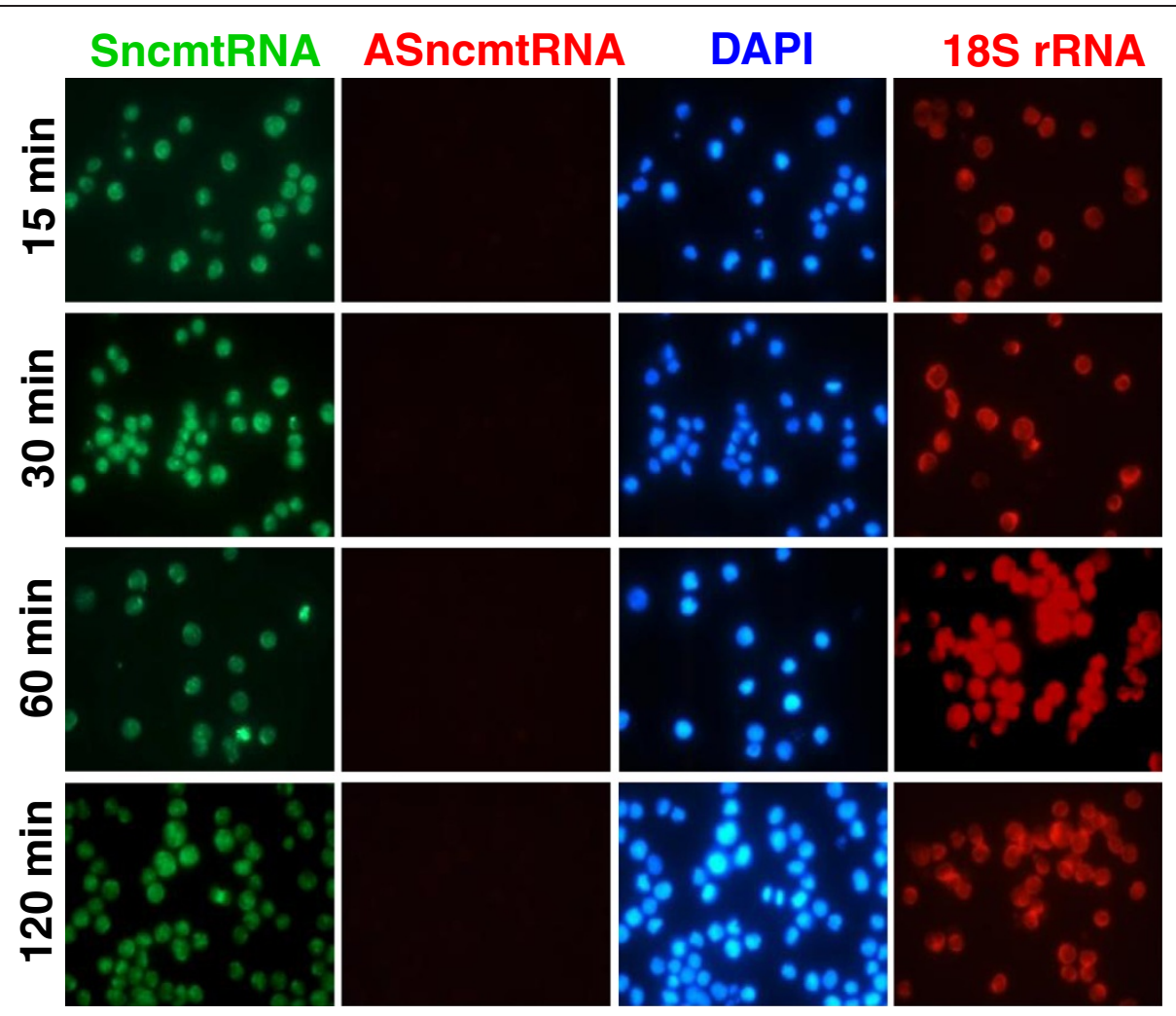

Figure 2 Hybridization kinetics on T-24 cells. After trypsinization, $10^{5}$ cells were collected by centrifugation at $200 \times g$ for 10 min and the pellet was subjected to S-FISH as indicated in Figure 1. Cells were hybridized simultaneously with probes complementary to the SncmtRNA and ASncmtRNAs for 15, 30, 60 and 120 min. Only the green signal corresponding to the expression of the SncmtRNA was observed, while expression of the ASncmtRNAs was down-regulated (red fluorescence). Cell distribution was revealed with DAPI staining (DAPI). The positive control corresponding to Texas red-labeled $18 \mathrm{~S}$ rRNA-probe was run in parallel (60x).

\section{S-FISH}

All the hybridization steps were performed in MaxiRecovery $^{\mathrm{TM}}$ tubes of $0.5 \mathrm{ml}$ (Axygen Scientific, US). After trypsinization (Invitrogen, Carlsbad, US), about $10^{5}$ cells were recovered by centrifugation at $200 \times g$ for $10 \mathrm{~min}$ at room temperature (RT). The cell pellet was resuspended in $100 \mu \mathrm{l} \mathrm{HCl} 0,2 \mathrm{~N}$ and incubated for $5 \mathrm{~min}$ at RT. Afterwards, the cell suspension was diluted with $400 \mu \mathrm{l}$ PBS (50 mM sodium phosphate, $150 \mathrm{mM} \mathrm{NaCl}$ and 2 mM EDTA, pH 9.0) and centrifuged again. The sediment was resuspended in $100 \mu$ l hybridization buffer (50\% formamide, $150 \mu \mathrm{g} / \mathrm{ml}$ herring sperm DNA, 4X SSC, $2 \mathrm{mM}$ EDTA) containing 0,5 $\mathrm{MM} 5^{\prime}$-Alexa fluor 488labeled probe P1 (5’ GTTCTTGGGTGGGTGTGGG 3’), complementary to the SncmtRNA and 0,05 $\mu \mathrm{M}$ each of two $5^{\prime}$ Texas Red-labeled probes P2 (5' GATAACAGCG CAATCCTATT $3^{\prime}$ ) and P3 (5' ACCGTGCAAAGGTAG CATAATCA $3^{\prime}$ ), complementary to the ASncmtRNAs. In addition, two negative controls corresponding to mismatch probes P5 for the SncmtRNA (MM: $5^{\prime}$ TTTATTT GATGAGTGTGAG 3'), labeled with Alexa fluor 488 and probe P6 for the ASncmtRNAs (MM: 5' GTAAAGATAG TATAATAATTTATTAATTAAATATA $3^{\prime}$ ), labeled with
Texas Red at the $5^{\prime}$ end. The labeled probes were obtained from Invitrogen (Carlsbad, CA, USA).

Hybridization was carried out for $15 \mathrm{~min}$ to $2 \mathrm{~h}$ at $37^{\circ} \mathrm{C}$. The final wash was performed by addition of four volumes of stringency buffer ( $2 \mathrm{X} \mathrm{SCC}+2 \mathrm{mM}$ EDTA) to the hybridization mix, incubated for $5 \mathrm{~min}$ at $37^{\circ} \mathrm{C}$ and finally centrifuged at $200 \times g$ for $10 \mathrm{~min}$. The supernatant was discarded and a small volume of approximately $20 \mu \mathrm{l}$ of the residual supernatant was left in the tube to resuspend the cells. The cells were finally stained in a solution of $1 \mu \mathrm{g} / \mathrm{ml}$ DAPI, deposited onto a positively charged slide (Thermo Scientific, US) and mounted in fluorescent medium (DAKO). Samples were analyzed by fluorescence microscopy on an Olympus BX-51 microscope under x600 magnification, with 300-600 ms exposition and results were documented with Q-capture Pro software. The positive hybridization control corresponded to a $5^{\prime}$-Texas Red-labeled probe complementary to $18 \mathrm{~S}$ rRNA (P4: $5^{\prime}$ AGTGGACTCATTCCAATTACA 3').

\section{Voided urine}

About 30-50 ml voided urine from male and female healthy donors was carried out in agreement with the 

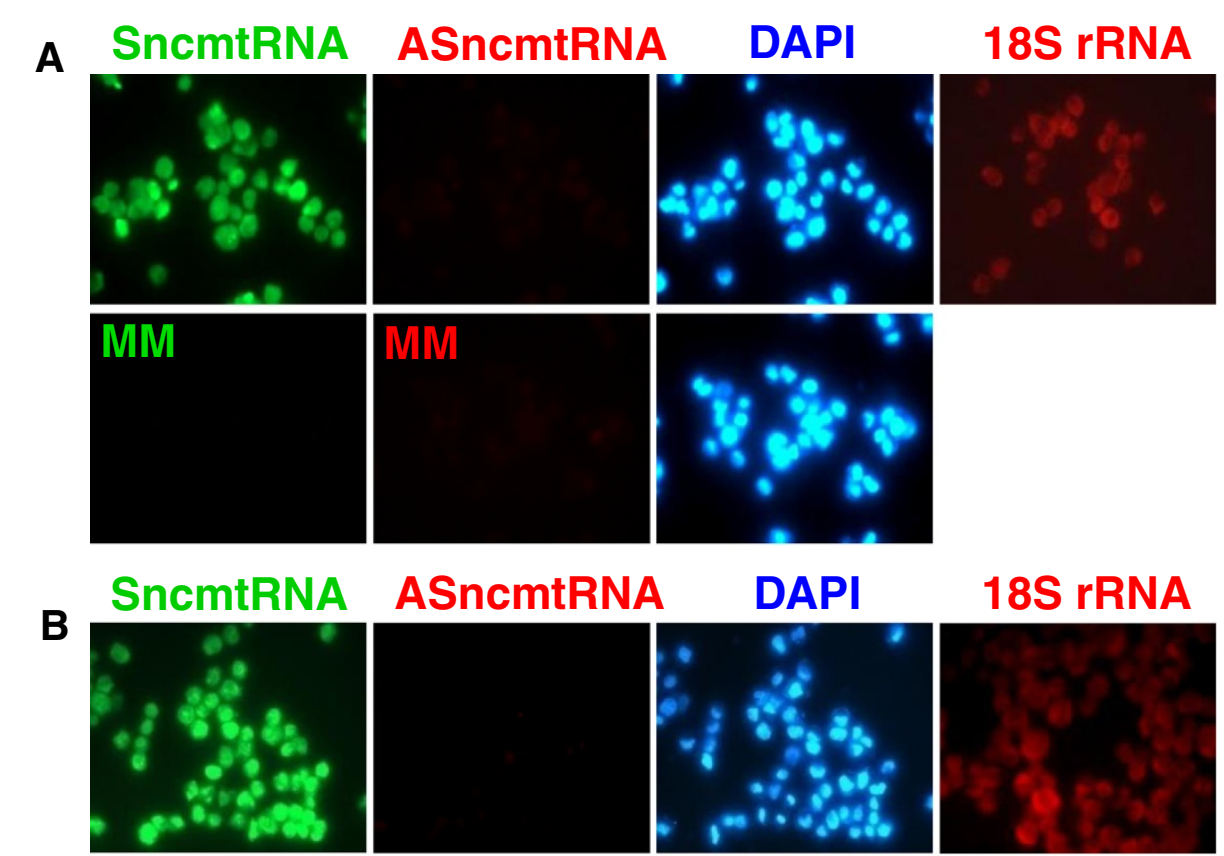

\section{S rRNA}
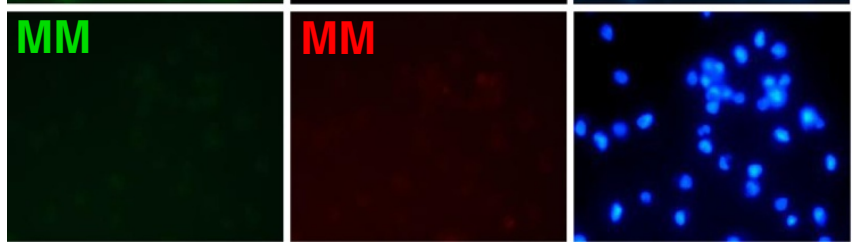

Figure 3 Stability of the mitochondrial ncRNAs in urine. A, $5 \times 10^{4} \mathrm{~T}-24$ cells were loaded in urine obtained from healthy donors and stored at $4^{\circ} \mathrm{C}$ for $24 \mathrm{~h}$. Cells were then recovered by centrifugation as described and subjected to S-FISH. Only the SncmtRNA (green fluorescence) was detectable, while probes for ASncmtRNAs (red fluorescence) showed absence of signal, as did the corresponding mismatch probes (MM, green or red). The cells were also counterstained with DAPI. A parallel sample was hybridized with a probe specific for the 18S rRNA (18S rRNA). B, DU-145 cells (prostate carcinoma) were subjected to the same S-FISH protocol. Notice that the cells only express the SncmtRNA. No fluorescent signal was obtained with the probes targeted to the ASncmtRNA or with MM probes.

ethical guidelines approved by the Ethical Committee of the Fundacion Ciencia para la Vida. The urine from healthy donors $(50 \mathrm{ml})$ was loaded with $5 \times 10^{4}$ to $1 \times 10^{5}$ T24 or DU-145 cells or PHA-stimulated lymphocytes and incubated at $4^{\circ} \mathrm{C}$ for $24 \mathrm{~h}$. The cells were sedimented by centrifugation at $700 \times g$ for $10 \mathrm{~min}$. The supernatant was discarded, leaving only $5 \mathrm{ml}$, which were transferred to a Kova tube (Hycor Biomedical Inc., US) and centrifuged again at $200 \times g$ for $10 \mathrm{~min}$ at RT. Most of the supernatant was discarded and the remnant ( 1ml) was transferred to a 0,5 $\mathrm{ml} \mathrm{Maxy-Recovery} \mathrm{tube}$ (Axygen Scientific, US), centrifuged at $200 \times \mathrm{g}$ and subjected to S-FISH as described above.

Twenty four patients diagnosed with $\mathrm{BC}$ were recruited at the Urology Unit of the Hospital Barros Luco Trudeau and Clinica Indisa (Santiago, Chile). The urine samples were obtained with informed consent under the Ethical Regulations of the Hospital Barros Luco Trudeau and Clinica Indisa and with the approval of the Ethic Committee of Fundacion Ciencia para la
Vida. The tumor biopsies were graded as reported [14] and the data are summarized in Table 1 . The firstmorning voided urine $(50 \mathrm{ml})$ was collected and stored at $4^{\circ} \mathrm{C}$ in a cooler and transported to the laboratory. The urine was centrifuged at $700 \times g$ for 10 min within $4 \mathrm{~h}$ after collection and S-FISH was performed as described above. Parallel samples were stained with hematoxylin.

\section{Results}

\section{Optimization of the S-FISH protocol}

A schematic representation of the S-FISH is shown in Figure 1. Briefly, cells were collected by centrifugation from cell culture or from biological fluids such as urine and blood, followed by permeabilization with $0.2 \mathrm{~N} \mathrm{HCl}$. After neutralization, the cells were recovered, hybridized with a set of probes labeled with fluorophores, washed and analyzed by fluorescence microscopy (see Methods). To determine the minimum hybridization time needed for the detection of the mitochondrial ncRNAs, T-24 cells (bladder carcinoma cell line) were subjected to 
S-FISH for different time periods. After 15 minutes of hybridization the fluorescent signal of the SncmtRNA was as strong and specific as longer hybridization times (Figure 2, SncmtRNA). The red signal corresponding to the ASncmtRNA was negative at any of the hybridization times tested confirming the tumor pattern of expression of T-24 cells (Figure 2, ASncmtRNAs). The $18 \mathrm{~S}$ rRNA used as positive control was expressed in all cells and the corresponding red signal was also independent of hybridization times ranging from 15 to $120 \mathrm{~min}$ (Figure 2, 18S rRNA, red). The same results were obtained with the bladder carcinoma cell line RT4 (unpublished data).

\section{Stability of the mitochondrial ncRNAs in urine}

An interesting model to test S-FISH was cancer cells obtained from voided urine of patients with BC. Since the stability of cells and their RNAs in urine is uncertain, we asked whether the urine would affect the stability of the SncmtRNA and the ASncmtRNAs from tumor cells and normal proliferating cells. Fresh urine of healthy donors was loaded with $5 \times 10^{4} \mathrm{~T}-24$ cells $/ \mathrm{ml}$ and maintained at $4^{\circ} \mathrm{C}$ for $24 \mathrm{~h}$. Cells were then recovered by centrifugation and subjected to S-FISH as described. Analysis of several fields indicated that all cells were positive for the SncmtRNA and the intensity of the fluorescent signal was comparable to that of fresh T-24 cells (compare Figures 2 and 3, SncmtRNA). The red hybridization signal corresponding to the ASncmtRNAs (Figure 3A, ASncmtRNAs) was negative and comparable to the negative green fluorescence of the mismatch (MM) probes for the sense transcript (Figure 3A, panels MM green) or the red fluorescence of the MM probe for the antisense transcripts (Figure 3A, panels MM red). The hybridization signal of $18 \mathrm{~S}$ rRNA was similar to that of fresh T-24 cells (Figure 3A, 18S rRNA). The same results were obtained with DU-145 cells (prostate carcinoma cell line) (Figure 3B).

In addition, we determined the stability of the SncmtRNA and the ASncmtRNAs in normal proliferating cells maintained in urine for $24 \mathrm{~h}$ at $4^{\circ} \mathrm{C}$. Isolated PBMC were activated with PHA and incubated in urine for $24 \mathrm{~h}$ previous to S-FISH. PHA-stimulated PBMCs showed a positive signal for both the SncmtRNA and the ASncmtRNAs (Figure 4A) confirming the expression pattern of normal proliferating cells [10]. On the other hand, the hybridization signals were negative with the MM probe to either the SncmtRNA (green fluorescence) and the ASncmtRNAs (red fluorescence) (Figure 3A, MM). Similarly, the same normal renal epithelial cells (DAPI staining) expressing the SncmtRNA were also expressing the ASncmtRNAs (Figure 4B). The green mismatch control was negative (Figure 4B).

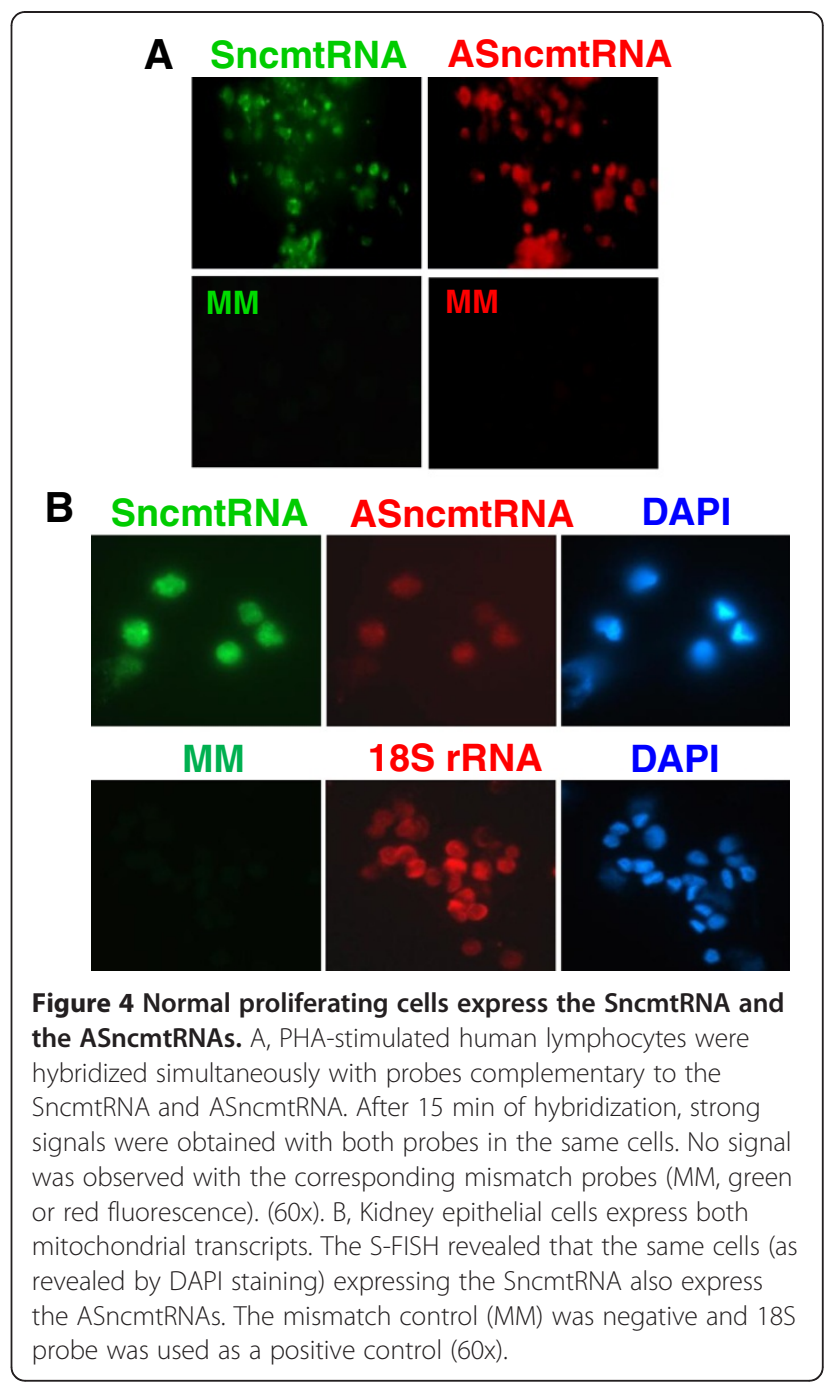

Detection of tumor cells in voided urine of patients with BC

Then we asked whether S-FISH can be applied to tumor cells present in voided urine obtained from 24 patients with $\mathrm{BC}$ diagnosed by cystoscopy and confirmed by biopsy. Table 1 show the grade and stage distribution of the samples. The cells were recovered from urine $4 \mathrm{~h}$ after collection and subjected to S-FISH as described before. Then, 25 fields of each sample at 40x magnification were analyzed and recorded. In all 24 urine samples, the $\mathrm{S}$-FISH detects cells expressing the SncmtRNA and down-regulate the ASncmtRNAs. As described before, this expression pattern corresponds to a cancer cells. Figure 5 illustrates S-FISH results obtained with urine cells recovered from four patients with BC. Samples A, $\mathrm{B}$ and $\mathrm{C}$ correspond to urine cells recovered from patients with grade $3 \mathrm{BC}$ (Figure 5). Samples D corresponds to cells obtained from patients with grade 2 (Figure 5). It is important to mention that the cellularity of samples $\mathrm{C}$ and D was low. Although the cellularity 


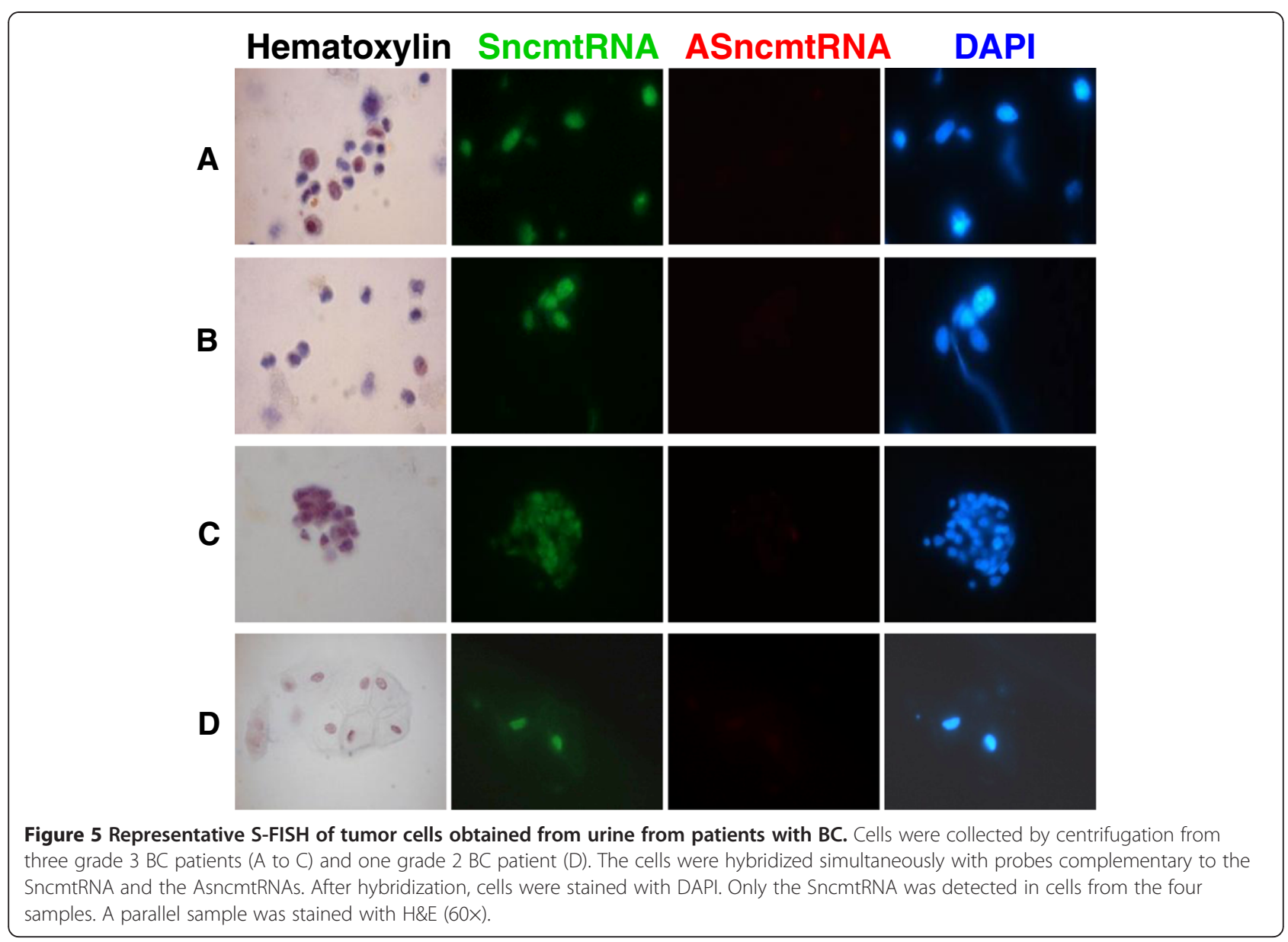

of grade $1 \mathrm{BC}$ was low, the S-FISH detected few cells that only express the SncmtRNA. In six urine samples obtained from the 15 healthy donors (Table 1) few cells were recovered. However, the hybridization signals indicate that the SncmtRNA and the ASncmtRNAs were down-regulated. The typical debris present in some urine samples did not interfere with the hybridization signal.

\section{Discussion}

FISH provides an important tool for conventional cytogenetics and evaluation of chromosomal abnormalities associated with several malignancies [15]. Some examples of chromosomal abnormalities are found in several diseases, such as BC [16-20], multiple myeloma [21-23], breast cancer [24], hematological malignancies [25-28] and lung cancer $[29,30]$ among others. Different types of tumor require specific sets of probes corresponding to particular chromosomal deletions/translocations characteristic of each cancer. Sokolova et al. reported the development of a FISH assay with high sensitivity and specificity for high grade $\mathrm{BC}$ using four labeled probes specific for the pericentromeric regions of chromosomes 3,7 and 17 and for the detection of the 9p21 deletion
[8]. These results were confirmed in later studies with a large cohort of BC patients [16-20] using several probes combined into a single multiprobe cocktail, to detect polysomy of chromosomes 3, 7 and 17 and homozygous deletion of $9 \mathrm{p} 21$ in the urine of $\mathrm{BC}$ patients (Urovysion, Abbot Molecular/Vysis, Des Plaines, IL). However, this test has low sensitivity for low-stage and low-grade tumors, which are the main group that recur [3].

The S-FISH assay described here is able to detect the differential expression of the SncmtRNA and the ASncmtRNAs in normal and cancer cells. This a simple protocol that was optimized in three steps including a single permeabilization step with $\mathrm{HCl}$, a short hybridization step and a brief washing that basically involves the dilution of the hybridization mix with stringency buffer (Figure 1). The protocol contains only three centrifugation steps in the same tube, minimizing the manipulation of cells and therefore maximizing RNA preservation and cell recovery. This test is reproducible and has been applied to other normal and tumor cell lines. Hybridization of normal proliferating cells (human umbilical vein endothelial cells, keratinocytes and melanocytes) reveals the expression of 
the SncmtRNA and the ASncmtRNAs. In other human tumor cell lines such as HeLa, 42/95 and SK-MEL-2 (melanoma), Jurkat and HL-60 (leukemia) and MDAMB-231 (breast carcinoma), S-FISH revealed expression of the SncmtRNA and down-regulation of the ASncmtRNA (unpublished data).

Moreover, S-FISH was able to detect cancer cells in urine from twenty four patients with $\mathrm{BC}$ and the results were independent of the grade of $\mathrm{BC}$ and the urine cellularity (see Figure 5). Taken together, the results suggest that the diagnostic test has a very high positive outcome independent of the grade and the amount of cells recovered from urine of patients with BC. In the urine from healthy donors, cells were recovered only from six out of fifteen samples and the S-FISH revealed absence of signal to both transcripts.

\section{Conclusions}

Taken together, this pilot study suggests that S-FISH could be used for detection and regular surveillance programs of patients with $\mathrm{BC}$. Interestingly, the results indicate that the exfoliated bladder tumor cells from low and high grade $\mathrm{BC}$ conserve the expression pattern observed in bladder cancer biopsies: expression of the SncmtRNA and down-regulation of the ASncmtRNAs. In summary, S-FISH may potentially be used as a noninvasive diagnostic test for bladder cancer. However, to validate the test, a large cohort of patients with lowgrade and high-grade neoplasms should be included together with other urological diseases such as glomerulonephritis, infections of the upper urinary track and other benign urinary track diseases.

\section{Competing interests}

Authors report no competing interests

\section{Authors' contributions}

$L O B, V B$ and $J V$ conceived the experimental plan, analyzed the data, and drafted the manuscript. $A R, V B, E L, V B, S G, R A, C L, C V, R F$, and JE carried out the experiments. RF, EL and VB reviewed the patients' history and pathological data. All authors read and approved the final manuscript.

\section{Acknowledgements}

Supported by Grant 1085210, FONDECYT, Millennium Scientific Initiative N ${ }^{\circ}$ P-77-09 F, Grants DI-20-11-I, Universidad Andrés Bello, Grant D0411338, FONDEF, the CCTE-PFB16 Program of Conicyt and Grant 12IDL4-13358, CORFO-INNOVA, Chile

\footnotetext{
Author details

${ }^{1}$ Andes Biotechnologies S.A. and Fundación Ciencia para la Vida, 7780272 Santiago, Chile. ${ }^{2}$ Departamento de Ciencia Biológicas, Facultad de Ciencias Biológicas, Universidad Andrés Bello, 8370146 Santiago, Chile. ${ }^{3}$ Facultad de Medicina, Universidad Andrés Bello, 8370146 Santiago, Chile. ${ }^{4}$ Urology Unit, Clínica Indisa, 7520440 Santiago, Chile. ${ }^{5}$ Urology Unit, Hospital Barros Luco Trudeau, 8900085 Santiago, Chile.
}

Received: 22 March 2012 Accepted: 7 December 2012

Published: 18 December 2012

\section{References}

1. Jemal A, Bray F, Center MM, Ferlay J, Ward E, Forman D: Global cancer statistics. CA Cancer J Clin 2011, 61:69-90.

2. Kaufman DS, Shipley WU, Feldman AS: Bladder cancer. Lancet 2009 374:239-249.

3. Van Tilborg AA, Bangma CH, Zwarthoff EC: Bladder cancer biomarkers and their role in surveillance and screening. Int J Urol 2009, 16:23-30.

4. Maier $U$, Simak R, Neuhold N: The clinical value of urinary cytology: 12 years of experience with 615 patients. J Clin Pathol 1995, 48:314-317.

5. Ross J, Cohen M: Ancilliary methods for the detection of recurrent urothelial neoplasia. Cancer 2000, 90:75-86.

6. Msaouel P, Koutsilieris M: Diagnostic value of circulating tumor cells detection in bladder and urothelial cancer: systematic review and metaanalysis. BMC Cancer 2011, 11:336-349.

7. Ecke T: Focus on urinary bladder cancer markers: a review. Minerva Urol Nefrol 2008, 60:237-246.

8. Sokolova IA, Halling KC, Jenkins RB, Burkhardt HM, Meyer RG, Seelig SA, King $W$ : The development of a multitarget, multicolor fluorescence in situ hybridization assay for the detection of urothelial carcinoma in urine. J Mol Diagn 2000, 2:116-123.

9. Villegas J, Burzio V, Villota C, Landerer E, Martinez R, Santander M, Martinez R, Pinto R, Vera MI, Boccardo E, Villa LL, Burzio LO: Expression of a novel non-coding mitochondrial RNA in human proliferating cells. Nucleic Acids Res 2007, 35:7336-7347.

10. Burzio VA, Villota C, Villegas J, Landerer E, Boccardo E, Villa LL, Martínez R, Lopez C, Gaete F, Toro V, Rodriguez X, Burzio LO: Expression of a family of noncoding mitochondrial RNAs distinguishes normal from cancer cells. Proc Natl Acad Sci USA 2009, 106:9430-9434

11. Villota C, Campos A, Vidaurre S, Oliveira-Cruz L, Boccardo E, Burzio VA, Varas M, Villegas J, Villa LL, Valenzuela PDT, Socias M, Roberts S, Burzio LO: Expression of mitocondrial ncRNAs is modulated by high risk HPV oncogenes. J Biol Chem 2012, 287:21303-21315.

12. Landerer E, Villegas J, Burzio VA, Oliveira L, Villota C, Lopez C, Restovic F, Martinez R, Castillo O, Burzio LO: Nuclear localization of the mitochondrial ncRNAs in normal and cancer cells. Cellular Oncol 2011, 34:297-305.

13. Dergunova NN, Bulycheva TI, Artemenko EG, Shpakova AP, Pegova AN, Gemjian EG, Dudnik OA, Zatsepina OV, Malashenko OS: A major nucleolar protein B23 as a marker of proliferation activity of human peripheral lymphocytes. Immunol Lett 2002, 83:67-72.

14. Babjuk M, Oosterlinck W, Sylvester R, Kaasinen E, Böhle A, Palou-Redorta J, Morgan Roupret M: EAU guidelines on non-muscle-invasive urothelial carcinoma of the bladder, the 2011 update. Eur Urol 2011, 59:997-1008.

15. Wolff DJ, Bagg A, Cooley LD, Dewald GW, Hirsch BA, Jacky PB, Rao KW, Rao PN: Guidance for fluorescence in situ hybridization testing in hematologic disorders. J Mol Diagn 2007, 9:134-143.

16. Halling KC, King W, Sokolova IA, Meyer RG, Burkhardt HM, Halling AC, Cheville JC, Sebo TJ, Ramakumar S, Stewart CS, Pankratz S, O'Kane DJ, Seelig SA, Lieber MM, Jenkins RB: A comparison of cytology and fluorescence in situ hybridization for the detection of urothelial carcinoma. J Urol 2000, 164:1768-1775

17. Kang JU, Koo SH, Jeong TE, Kwon KC, Park JW, Jeon CH: Multitarget fluorescence in situ hybridization and melanoma antigen genes analysis in primary bladder carcinoma. Cancer Genet Cytogenet 2006, 164:32-38.

18. Kipp BR, Tanasescu M, Else TA, Bryant SC, Karnes RJ, Sebo TJ, Halling KC: Quantitative fluorescence in situ hybridization and its ability to predict bladder cancer recurrence and progression to muscle-invasive bladder cancer. J Mol Diagn 2009, 11:148-154.

19. Colucci G, Floege J, Schena FP: The urinary sediment beyond light microscopical examination. Nephrol Dial Transplant 2006, 21:1482-1485

20. Meiers I, Singh H, Hossain D, Lang K, Liu L, Qian J, Verhest AP, Bostwick DG: Improved filter method for urine sediment detection of urothelial carcinoma by fluorescence in situ hybridization. Arch Pathol Lab Med 2007, 131:1574-1579.

21. Stewart AK, Fonseca R: Review of molecular diagnostics in multiple myeloma. Expert Rev Mol Diagn 2007, 7:453-459.

22. Bryce AH, Ketterling RP, Gertz MA, Lacy M, Knudson RA, Zeldenrust S, Kumar S, Hayman S, Buadi F, Kyle RA, Greipp PR, Lust JA, Russell S, Rajkumar SV, Fonseca $R$, Dispenzieri A: Translocation $t(11 ; 14)$ and survival of patients with light chain (AL) amyloidosis. Haematologica 2009, 94:380-386. 
23. Avet-Loiseau H, Soulier J, Fermand JP, Yakoub-Agh: IFM and MAG groups. Impact of high-risk cytogenetics and prior therapy on outcomes in patients with advanced relapsed or refractory multiple myeloma treated with lenalidomide plus dexaméthasone. Leukemia 2010, 24:623-628.

24. Pauletti G, Godolphin W, Press MF, Slamon DJ: Detection and quantitation of HER-2/neu gene amplification in human breast cancer archival material using fluorescence in situ hybridization. Oncogene 1996, 13:63-72.

25. Escudier SM, Pereira-Leahy JM, Drach JW, Weier HU, Goodacre AM, Cork MA, Trujillo JM, Keating MJ, Andreeff M: Fluorescent in situ hybridization and cytogenetic studies of trisomy 12 in chronic lymphocytic leukemia. Blood 1993, 81:2702-2707.

26. Hagemeijer A, Buijs A, Smit E, Janssen B, Creemers GJ, Van der Plas D, Grosveld G: Translocation of BCR to chromosome 9: a new cytogenetic variant detected by FISH in two Ph-negative, BCR-positive patients with chronic myeloid leukemia. Genes Chromosomes Cancer 1993, 8:237-245.

27. Zanardi A, Bandiera D, Bertolini F, Corsini CA, Gregato G, Milani P, Barborini E, Carbone R: Miniaturized FISH for screening of onco-hematological malignancies. Biotechniques 2010, 49:497-504.

28. Nelson B, Gupta R, Dewald G, Paternoster S, Rosen S, Peterson L: Chronic lymphocytic leukemia FISH impact on diagnosis panel. American J Clinical Pathol 2007, 128:323-332.

29. Jiang F, Caraway NP, Nebiyou Bekele B, Zhang HZ, Khanna A, Wang H, Li R, Fernandez RL, Zaidi TM, Johnston DA, Katz RL: Surfactant protein A gene deletion and prognostics for patients with stage I non-small cell lung cancer. Clin Cancer Res 2005, 11:5417-5424.

30. Li R, Liu Z, Fan T, Jiang F: A novel multiple FISH array for the detection of genetic aberrations in cancer. Lab Invest 2006, 86:619-627.

doi:10.1186/1471-2490-12-37

Cite this article as: Rivas et al:: Determination of the differential expression of mitochondrial long non-coding RNAs as a noninvasive diagnosis of bladder cancer. BMC Urology 2012 12:37.

\section{Submit your next manuscript to BioMed Central and take full advantage of:}

- Convenient online submission

- Thorough peer review

- No space constraints or color figure charges

- Immediate publication on acceptance

- Inclusion in PubMed, CAS, Scopus and Google Scholar

- Research which is freely available for redistribution 